\title{
Genome-wide association study confirm major QTL for backfat fatty acid composition on SSC14 in Duroc pigs
}

\author{
Maren van Son ${ }^{1 *} \mathbb{D}$, Eli Gjerlaug Enger ${ }^{1}$, Harald Grove², Roger Ros-Freixedes ${ }^{3,4}$, Matthew P. Kent ${ }^{2}$, Sigbjørn Lien² \\ and Eli Grindflek'
}

\begin{abstract}
Background: Fatty acid composition contributes importantly to meat quality and is essential to the nutritional value of the meat. Identification of genetic factors underlying levels of fatty acids can be used to breed for pigs with healthier meat. The aim of this study was to conduct genome-wide association studies (GWAS) to identify QTL regions affecting fatty acid composition in backfat from the pig breeds Duroc and Landrace.

Results: Using data from the Axiom porcine $660 \mathrm{~K}$ array, we performed GWAS on 454 Duroc and 659 Landrace boars for fatty acid phenotypes measured by near-infrared spectroscopy (NIRS) technology (C16:0, C16:1n-7, C18:0, C18:1n-9, C18:2n-6, C18:3n-3, total saturated fatty acids, monounsaturated fatty acids and polyunsaturated fatty acids). Two QTL regions on SSC4 and SSC14 were identified in Duroc for the de novo synthesized fatty acids traits, whereas one QTL on SSC8 was detected in Landrace for C16:1n-7. The QTL region on SSC14 has been reported in previous studies and a putative causative mutation has been suggested in the promoter region of the SCD gene. Whole genome re-sequencing data was used for genotype imputation and to fine map the SSC14 QTL region in Norwegian Duroc. This effort confirms the location of the QTL on this chromosome as well as suggesting other putative candidate genes in the region. The most significant single nucleotide polymorphisms (SNPs) located on SSC14 explain between 55 and 76\% of the genetic variance and between 27 and 54\% of the phenotypic variance for the de novo synthesized fatty acid traits in Norwegian Duroc. For the QTL region on SSC8 in Landrace, the most significant SNP explained 19\% of the genetic variance and 5\% of the phenotypic variance for C16:1n-7.
\end{abstract}

Conclusions: This study confirms a major QTL affecting fatty acid composition on SSC14 in Duroc, which can be used in genetic selection to increase the level of fatty acid desaturation. The SSC14 QTL was not segregating in the Landrace population, but another QTL on SSC8 affecting C16:1n-7 was identified and might be used to increase the level of desaturation in meat products from this breed.

Keywords: Fatty acid composition, GWAS, QTL mapping, Fine mapping, Pigs

\section{Background}

The fatty acid composition of meat is important for its nutritional properties influencing human health, while also affecting the technical and sensory quality of meat products [1]. Fatty acids may be divided into two groups based on whether they are derived directly from the diet (essential) or whether they can be synthesized de novo through lipogenesis (non-essential). Fatty acids up to 16

\footnotetext{
* Correspondence: maren.van.son@norsvin.no

${ }^{1}$ Norsvin SA, Storhamargata 44, 2317, Hamar, Norway

Full list of author information is available at the end of the article
}

carbons in length are synthesized de novo and some are, together with fatty acids from the diet, further elongated into fatty acids with more than 18 carbons in length. These saturated fatty acids (SFA; C16:0 and C18:0) can then be desaturated to monounsaturated fatty acids (MUFA; C16:1n-7 and C18:1n-9). Polyunsaturated fatty acids (PUFA), with two or three double bounds, are obtained from the diet [1].

Fatty acid composition has received attention due to public health concerns related to evidence that saturated fat can increase the amount of cholesterol in our blood, which 
may in turn increase the risk of heart disease and stroke. Healthier fat can be produced by increasing the levels of MUFA and PUFA, lowering levels of SFA and decreasing the ratio of $n-6 / n-3$ PUFA [1-4]. The healthiness of the different types of fat is however debated, and some studies show that SFA is not as dangerous as previously claimed $[5,6]$. Fatty acid composition is also important for meat quality, and high levels of PUFA negatively impacts meat quality traits such as oxidative stability and flavor [2]. Increasing the content of the MUFA C18:1n-9, however, could improve both organoleptic and technological qualities as well as nutritional properties $[7,8]$.

Previous studies in Landrace and Duroc have obtained high heritabilities for the fatty acids C16:0, C16:1n-7, C18:0, C18:1n-9, C18:2n-6 and C18:3n-3 [9]. The heritability estimates, ranging from 0.25 to 0.67 , are in agreement with other studies $[10,11]$, suggesting that breeding pigs for favorable fatty acid composition is possible.

Numerous studies in different pig populations have identified quantitative trait loci (QTLs) for fatty acid composition in pig [12-25]. Significant QTLs have been identified on all the pig chromosomes and some are shared across breeds, populations and tissues or muscle groups. Candidate gene studies have been conducted to possibly identify functional mutations underlying the differences in fatty acid composition. On SSC8, promising results have been found for microsomal triglyceride transfer protein (MTTP), which is involved in lipoprotein assembly [26], and ELOVL fatty acid elongase 6 (ELOVL6), which catalyzes the elongation of C12-16 fatty acids to $C 18$ [17, 27]. On SSC14, the stearoyl-CoA desaturase (SCD) gene strikes out as a potent positional candidate gene for fat desaturation. The gene has been investigated in several studies [28-32] and a putative causal variant in the promoter region has been identified [28].

The aim of this study was to detect genomic regions controlling fatty acid composition in backfat from the Norwegian Duroc and Landrace breeds. For this purpose, six different fatty acid were measured in backfat from boars at a laboratory by NIRS technology, specifically C16:0, C16:1n-7, C18:0, C18:1n-9, C18:2n-6, C18:3n-3. Moreover, total SFA, MUFA and PUFA were included in the analyses. Boars were genotyped using the Axiom porcine $660 \mathrm{~K}$ SNP array (Affymetrix Inc.) and a GWAS was conducted. Whole genome re-sequencing data from related boars was used for subsequent fine mapping.

\section{Methods}

\section{Animals}

Animals from three groups of boars were included in this study. Group 1 was composed of 454 Duroc and 659 Landrace boars born in 2011 and 2012 from Norsvin's boar testing station. Animals were kept in single-breed groups of 12 pigs per pen and fed ad libitum on conventional concentrates. The diet contained 161 and $136 \mathrm{~g}$ digestible protein, and 9.68 and $9.50 \mathrm{MJ}$ net energy/ $\mathrm{kg}$ before and after $50 \mathrm{~kg}$ live weight, respectively, with one month of mixing the two feeds to facilitate the feed change. Major feedstuff compounds were barley, oats, peas, soy meal extract and rendering (animal) fat. Average fat percentage of the feeds was $2.6 \%$, with the following fatty acid profile (in percentage of total fatty acids): C16:0, 22.2\%; C16:1n-7, 1.0\%; C18:0, 8.9\%; C18:1n-9, 24.2\%; C18:2n-6, 35.4\%; C18:3n-3, 4.1\%; total SFA, 33.2\%; total MUFA, 26.3\%; and total PUFA, 39.8\%. The average start and end weight of the pigs for the test was 35 and $120 \mathrm{~kg}$ live weight, respectively. Slaughter weight can vary some as the boars are waiting for selection or not, and this was corrected for using a simple generalized linear model (GLM). The boars in this test were all selection candidates to be elite boars for artificial insemination (AI). Nonselected boars goes to slaughter and are available for meat quality assessment, including the ones in this study. The sacrifice procedure is described in more detail in Gjerlaug-Enger et al. [33] and was in compliance with national guidelines. For the animals in this study, phenotypes in terms of fatty acid composition and genotypes from the Illumina porcine $60 \mathrm{~K} \mathrm{SNP}$ chip (Illumina) were available.

Group 2 animals included 140 Duroc and 207 Landrace boars that were genotyped using the Axiom porcine $660 \mathrm{~K}$ array. These animals were frequently used AI boars between 2010 and 2015 and were close relatives to Group 1 animals. $60 \mathrm{~K}$ genotypes were also available from all the Group 2 animals. Additional $60 \mathrm{~K}$ genotypes were available for several thousand relatives making imputation of genotypes from 60 to $660 \mathrm{~K}$ feasible.

Whole genome re-sequencing data was available from Group 3 animals constituting 23 Duroc and 24 Landrace boars. These boars were frequently used as AI boars during the years 2010 to 2013 and overlap with Group 2 animals (6/10 of the Duroc/Landrace Group 3 animals were also Group 2 animals). $60 \mathrm{~K}$ genotypes were also available for the Group 3 animals.

\section{Fatty acid measurements}

Subcutaneous fat samples were collected from half-sib tested animals after slaughter at Animalia's pilot plant (the Norwegian Meat and Poultry Research Centre, Oslo, Norway). The samples were collected from the area by the last thoracic vertebrae, stored at $-40{ }^{\circ} \mathrm{C}$ and thereafter prepared at the BioBank AS (Hamar, Norway). Before analyses, they were thawed and minced, followed by isolation of total lipids using a microwave fat melting technique [34]. A XDS near-infrared rapid content analyzer (FOSS NIRSystems, Hillerød, Denmark) was used to obtain transflection spectra of the total lipids from the samples. The fatty acids measured were palmitic (C16:0), palmitoleic (C16:1n-7), stearic (C18:0), 
oleic (C18:1n-9), linoleic (C18:2n-6) and $\alpha$-linolenic (C18:3n-3). Each was measured as percentage of total fatty acids in backfat and expressed as grams/100 grams. Low percentage fatty acids were not included due to concerns over accuracy for low concentration measurements. In total, the above-mentioned fatty acids accounted for $95 \%$ of the total fatty acid content. Finally, total percentages of SFA, MUFA and PUFA were measured according to methods and calibration curves described in previous publications [9, 35].

\section{Genotyping}

Genomic DNA was extracted from ear biopsies using BioSprint DNA Kit (Qiagen, Hilden, Germany). DNA concentration and quality was measured using a NanoDrop ND-1000 spectrophotometer (NanoDrop Technologies, DE, USA). Genome wide SNP genotyping was performed using either the Axiom porcine $660 \mathrm{~K}$ array from Affymetrix (Affymetrix Inc., Santa Clara, CA, USA) [36], which contains assays for 658,692 SNPs, or using the Illumina porcine $60 \mathrm{~K}$ SNP chip containing 62,163 SNPs (Illumina, San Diego, USA) [37]. For the Affymetrix array, genotypes were assigned using Axiom Analysis Suite following the best practices protocol recommendations. Genotypes from the Illumina array were generated using GenomeStudio software. SNPs were filtered based on call rate $>0.97$ and minor allele frequency $(\mathrm{MAF})>0.01$. All SNP positions were based on Sscrofa genome build 10.2 [38].

\section{Imputation from $60 \mathrm{~K}$ to $660 \mathrm{~K}$}

After removing $660 \mathrm{~K}$ SNPs not passing quality scores, we were left with 398,809 SNPs for 140 Duroc boars and 413,094 SNPs for 207 Landrace boars. The 5855 Duroc and 10,976 Landrace pigs genotyped on the $60 \mathrm{~K}$ SNP chip were imputed to $660 \mathrm{~K}$ using the available $660 \mathrm{~K}$ genotyped pigs as reference. The number of filtered, high quality $60 \mathrm{~K}$ SNPs overlapping with $660 \mathrm{~K}$ used for this imputation was 29,433 for Duroc and 30,971 for Landrace. SNPs that were shared between the arrays were checked for matching genotypes and allele frequencies. The imputation was performed separately for each breed. The software used was FImpute v.2.2 [39] and we used default settings together with the complete pedigree as additional information.

\section{Genome wide association analyses}

GWAS analyses were run using the $\mathrm{R}$ v.3.2.4 package GenABEL v.1.8-0 [40, 41]. Phenotypes included the six fatty acids and three combined traits described above and they were pre-corrected for day of slaughter. For each trait, a test was run using the polygenic function, which combines the Family Based Score Test for Association (FASTA) with a kinship matrix of relatedness estimated from genotyped SNPs using the identity-by-state function. The $p$-values were corrected for genomic control by dividing the observed test statistic by a calculated genomic inflation factor, and a $p$-value of $1.0^{-6}$ was required for genome-wide significance. Manhattan plots were generated using the $\mathrm{R}$ package qqman v.0.1.2 [41, 42]. Genetic parameters were estimated using ASReml v.3.0 [43] by fitting SNP as a fixed effect and animal ID as a random effect. The fraction of genetic and phenotypic variance explained by each SNP for each phenotype was calculated as $2 p(1-p) \alpha^{2}$, divided by the additive genetic variance and phenotypic variance, respectively [44]. Here $\mathrm{p}$ is the frequency of the A allele in a SNP with the two alleles A and a, and $\alpha$ is the allele substitution effect. The Haploview software v.4.2 [45] was used with phased data to calculate linkage disequilibrium (LD) as expressed by $\mathrm{r}^{2}$.

\section{Sequencing data analyses}

Genomic DNA from 23 Duroc and 24 Landrace boars was extracted from blood or semen samples using the MagAttract DNA Blood Midi M48 protocol on the BioRobot M48 (Qiagen, Hilden, Germany). Whole genome DNA sequencing was performed by a commercial provider using an Illumina GAII platform generating $2 \times$ 100 bp reads according to manufactures' instructions.

Reads were quality checked using FastQC v.0.10.1 (Babraham Bioinformatics, UK) and trimmed using Sickle with the options paired end, a length for trimming of 50 and otherwise default settings [46]. BWA-aln v.0.7.5 was used to align the reads to Sscrofa build 10.2 [47] using default settings. Duplicates were marked and files sorted with SAMtools v.0.1.19 [48] and SNPs in the most significant QTL region were detected using FreeBayes v.1.0.2 [49]. For the initial detection of putative variants, a minimum of $2 \mathrm{x}$ read coverage was set. The detected variants were filtered using VCFtools v.0.1.14 [50] and SAMtools bcftools v.1.3 [47] by the following criteria: minimum $2 \mathrm{x}$ read coverage for a new allele with both reference and alternate allele present on both strands, minimum quality score of 25 , and a mapping quality of $>10$ for both alleles at a SNP position. A distance of at least 4 and $10 \mathrm{bp}$ to the next insertion/deletion (indel) was applied for SNPs and indels, respectively, and variants with more than one unique non-reference allele were removed. The variants were also filtered on sequencing depth because such variants are likely to be located in duplicated regions and be the result of misalignment. The Ensembl Variant Effect Predictor (VEP) software was applied to predict the effect of the detected SNPs [51]. The whole genome resequencing generated 10.1 billion paired-end reads with coverage ranging from 9-17X across the entire genome. A total of 48,346 variants were detected in the $8 \mathrm{Mb}$ QTL region in the Duroc pigs. After filtering, 18,252 SNPs were left in the reference panel for imputation. 


\section{Imputation from $660 \mathrm{~K}$ to sequence}

Genotype likelihoods outputted from FreeBayes were used to impute sporadic missing genotypes in the sequenced animals (reference panel) using Beagle v.4.1 [52, 53]. The same software was then used to phase the reference panel. Prior to imputation, the target panel $(660 \mathrm{~K})$ was compared to the reference panel using conform-gt [53] to exclude target variants without a corresponding reference panel record and to adjust target records to match the chromosome strand and allele order in the reference panel. The $660 \mathrm{~K}$ panel genotypes within the QTL region were imputed to the sequence based genotypes of a population of 5996 Duroc animals. After imputation, SNPs with a $\mathrm{MAF}<0.01$ were removed before further analyses. Conform-gt removed 9 of the $660 \mathrm{~K}$ SNPs due to unknown strand issues and 104 because they were not in the reference panel, leaving 1151 of the 660 K SNPs in the region for imputation. They were combined with the 18,252 SNPs in the reference panel and after filtering for MAF < $0.01,13,565$ SNPs were left for sequence based association analyses and representing one SNP every 590 bp on average. The newly detected and filtered SNPs have been submitted to dbSNP [54].

\section{Sequence based association analyses}

Sequence based association analyses were run in ASReml fitting each SNP as a fixed effect in the model. The phenotypes were the same as in the GWAS analyses, and a pedigree-based relationship matrix was fitted. The $p$ values were corrected for multiple testing using the Bonferroni correction method as implemented in the stats package v.3.2.4 in R v.3.2.4 [41].

\section{Results}

A GWAS was conducted using the Axiom porcine $660 \mathrm{~K}$ array to identify loci associated with fatty acid composition in backfat of Duroc and Landrace boars. Descriptive statistics for the fatty acid phenotypes, summarized in Table 1, show that the two breeds differ with respect to fatty acid composition, with Landrace typically having higher levels of total MUFA, whereas Duroc on average have higher levels of total SFA [9]. The GWAS detect highly significant QTLs for the six de novo synthesized fatty acids (C16:0, C16:1n-7, C18:0, C18:1n-9, SFA and MUFA) on SSC4 and SSC14 in Duroc (Fig. 1 and Additional file 1). The QTL results are summarized in Table 2. The most significant results on SSC14 in Duroc were in the interval from 117.6 to 124.6 Mb. The most significant SNP for C16:0 and SFA was rs318243431, which is located at position $120,643,956$ bp on SSC14 within intron 3 of the carboxypeptidase $N$ subunit 1 (CPN1) gene. The most significant SNP for C18:0, C18:1n-9 and MUFA was rs318695446, which is located at position 121,401,766 bp on SSC14, in an intergenic region between the genes $S C D$ and paired box 2
Table 1 Summary statistics for fatty acid composition

\begin{tabular}{lllllll}
\hline Trait & Breed & $\mathrm{n}$ & Mean & \multicolumn{1}{l}{ SD } & \multicolumn{1}{c}{ Min } & Max \\
\hline C16:0 & Landrace & 659 & 19.56 & 0.87 & 16.69 & 22.26 \\
& Duroc & 454 & 20.62 & 0.75 & 17.77 & 22.84 \\
C16:1n-7 & Landrace & 659 & 2.40 & 0.20 & 1.52 & 3.08 \\
& Duroc & 454 & 2.03 & 0.26 & 1.30 & 2.89 \\
C18:0 & Landrace & 659 & 11.61 & 1.00 & 8.83 & 16.98 \\
& Duroc & 454 & 14.49 & 1.64 & 9.96 & 18.42 \\
C18:1n-9 & Landrace & 659 & 42.38 & 1.54 & 34.43 & 46.91 \\
& Duroc & 454 & 39.97 & 2.24 & 34.04 & 42.00 \\
C18:2n-6 & Landrace & 659 & 15.90 & 1.74 & 10.71 & 23.61 \\
& Duroc & 454 & 15.69 & 1.61 & 10.95 & 20.64 \\
C18:3n-3 & Landrace & 659 & 1.75 & 0.20 & 1.04 & 2.51 \\
& Duroc & 454 & 1.65 & 0.20 & 0.90 & 2.48 \\
PUFA & Landrace & 659 & 19.57 & 2.03 & 13.75 & 27.98 \\
& Duroc & 454 & 19.16 & 1.83 & 13.37 & 24.53 \\
SFA & Landrace & 659 & 33.54 & 1.55 & 29.58 & 40.07 \\
& Duroc & 454 & 37.29 & 2.20 & 29.47 & 42.17 \\
MUFA & Landrace & 659 & 45.92 & 1.67 & 36.52 & 50.77 \\
& Duroc & 454 & 42.79 & 2.60 & 35.95 & 50.22 \\
& L & & 459 &
\end{tabular}

For each breed, mean, standard deviation, minimum and maximum of the different fatty acids are presented ( $n=$ number of animals)

(PAX2). For $\mathrm{C} 16: 1 \mathrm{n}-7$, the most significant SNP was rs340458768, which is located at SSC14 position $121,565,853$ bp and falls within intron 8 of $P A X 2$. There were 21 significant SNPs on SSC4 for all the de novo synthesized fatty acid traits and they are located at 63.8563.99 Mb. In Landrace, a QTL on SSC8:120.5-121.4 Mb showed significant association for C16:1n-7 (Fig. 2, Table 2 and Additional file 2). The most significant SNP of this QTL is located in an intergenic region between two uncharacterized protein coding genes. No significant results were found for any of the other traits in Landrace. Moreover, no significant associations were found for the essential fatty acids (C18:2n-6, C18:3n-3 and total PUFA) in either Duroc or Landrace. Allele substitution effects and the proportion of explained genetic and phenotypic variance for the most significant SNPs are given in Table 3. On SSC14 in Duroc, the most significant SNP was shown to explain between 55 and $76 \%$ of the genetic variance and between 27 and $54 \%$ of the phenotypic variance for the different traits. For the most significant QTL in this study, region 117-124 Mb on SSC14, box plots were made to visualize the mean phenotypic differences per genotype class for the most significant SNP (Fig. 3). The phenotypic difference is clear when comparing the three genotypes and indicates an additive genetic effect. Considering the human health perspective and a goal of reduced SFA and increased MUFA, the favorable allele for all traits in Duroc is the least frequent within the population. 
C16:0

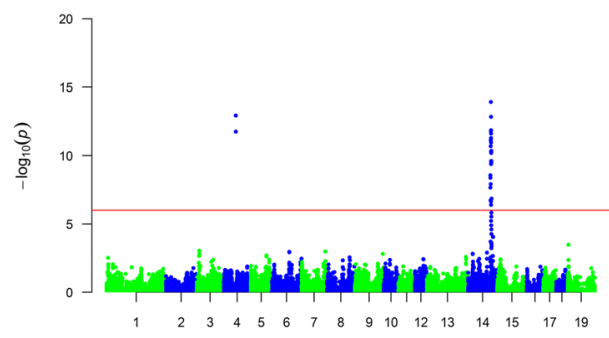

Chromosome

C18:0

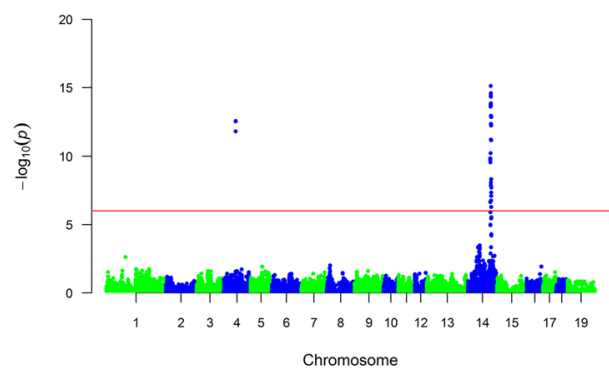

C18:2

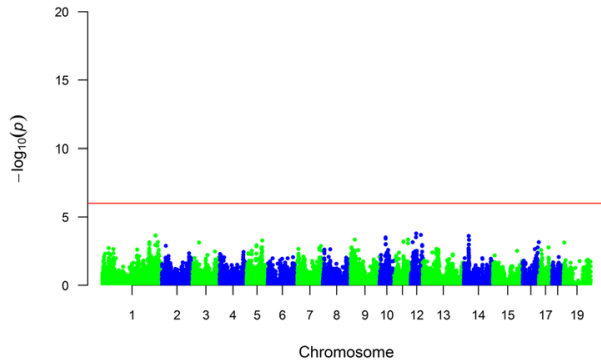

SFA

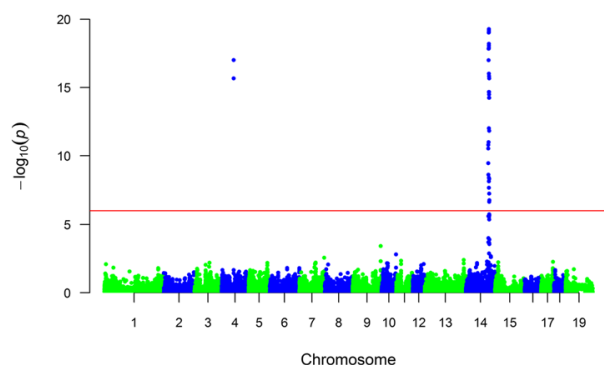

PUFA

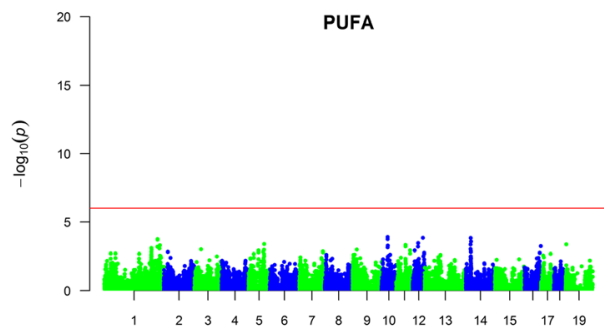

Chromosome
C16:1

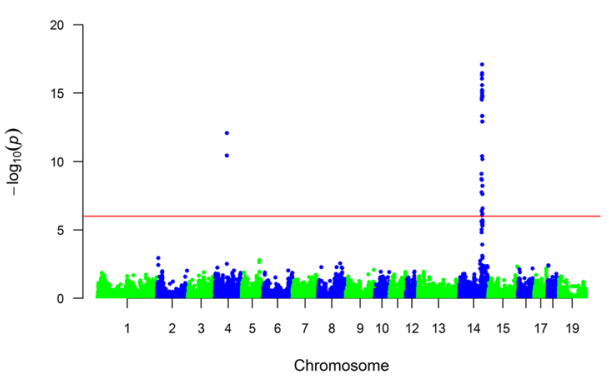

C18:1

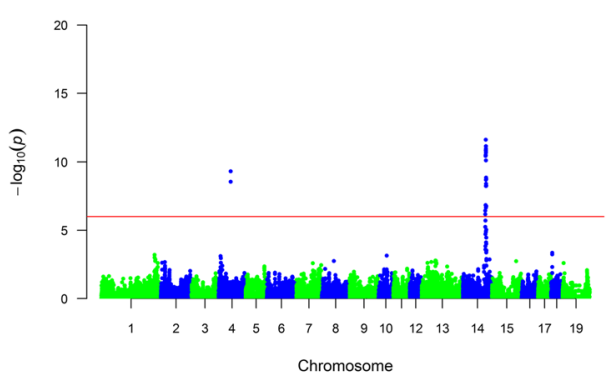

C18:3

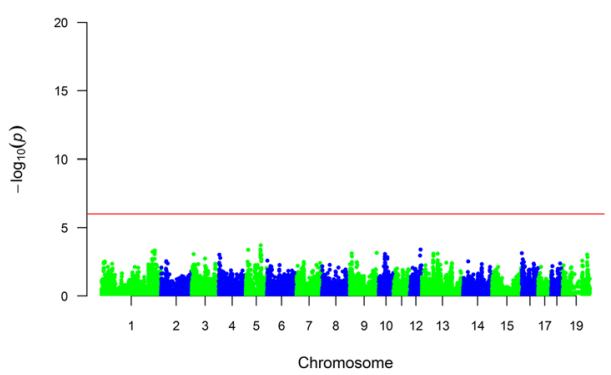

MUFA

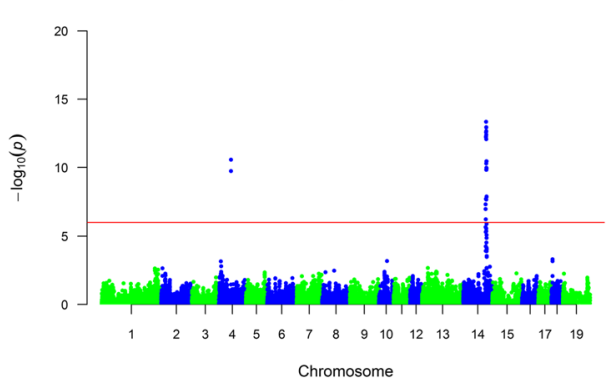

Fig. 1 Manhattan plots showing genome-wide association results for fatty acid composition in Duroc. SNPs are plotted on the x-axis according to their position on each chromosome against statistical association with these traits on the $y$-axis. The horizontal line indicates genome-wide significance $\left(p<1.0^{-6}\right)$ 
Table 2 QTL regions identified

\begin{tabular}{lllll}
\hline SSC & Position (Mb) & Breed & Trait & \#significant SNPs \\
\hline 4 & $63.85-63.99$ & Duroc & C16:0 & 21 \\
& & Duroc & C16:1n-7 & 21 \\
& & Duroc & C18:0 & 21 \\
& & Duroc & C18:1n-9 & 21 \\
& & Duroc & SFA & 21 \\
& & Duroc & MUFA & 21 \\
14 & $120.5-121.4$ & Landrace & C16:1n-7 & 23 \\
14 & $117.6-124.6$ & Duroc & C16:0 & 624 \\
& $117.6-124.6$ & Duroc & C16:1n-7 & 648 \\
& $117.6-124.6$ & Duroc & C18:0 & 680 \\
& $118.1-123.5$ & Duroc & C18:1n-9 & 545 \\
& $117.6-124.6$ & Duroc & SFA & 689 \\
& $118.1-123.5$ & Duroc & MUFA & 566
\end{tabular}

Traits are listed with significant QTL regions (SSC and position (Mb)) and the number of significant SNPs within each QTL region $\left(p<1.0^{-6}\right)$

Whole genome re-sequencing data from Duroc $(n=$ 23) boars was used to impute sequence-based genotypes in the $8 \mathrm{Mb}$ QTL region on SSC14 in Duroc. The sequence based association study confirmed the findings of a highly significant QTL for the de novo synthesized fatty acids on SSC14 and revealed moderate to high LD in the QTL region at 120-122 Mb (Fig. 4). All significant SNPs in this region are given in Additional file 3. The most significant SNP for C16:0 was found at position 120,952,148 which is intergenic between the genes polycystin 2 like 1, transient receptor potential cation channel (PKD2L1) and SCD. For C16:1n-7 and MUFA, 21 highly significant SNPs, being in almost complete LD with each other, were located in the region at 121.56121.60 Mb. The SNPs are positioned in introns and downstream of $P A X 2$, and in the intergenic region between PAX2 and the semaphoring 4G (SEMAG4) gene. The most significant SNP for C18:0 and SFA was at $120,643,956 \mathrm{Mb}$ which is within intron 3 of CPN1. For C18:1n-9 the most significant SNPs, seven in total LD, were located at $120,29-120.30 \mathrm{Mb}$, which is in intron 57 of the gene ectonucleoside triphosphate diphosphohydrolase 7 (ENTPD7).

The whole genome re-sequencing data was also used to examine the suggested causative variant rs80912566 in the promotor of SCD [28]. While all three genotypes were found in the Duroc boars, the Landrace boars were fixed for the $\mathrm{T}$ allele, being the allele associated with higher fat desaturation. The LD between the SCD genotype and the most significant SNPs in Duroc rs318695446, rs318243431 and $r s 340458768$ was $r^{2}=0.82,0.80$ and 0.95 , respectively. Based on findings of putative misplaced SNPs in a previous study [15], we also inspected the LD between the significant SNPs on SSC4 and SSC14, and found that the degree of LD between SNPs on these chromosomes was in the range of $r^{2}=0.85-1.0$.

\section{Discussion}

Knowledge of the fatty acid composition of pork can be used to manipulate levels of different fatty acids and thereby produce pigs with healthier meat [2]. In the present study, we conducted GWAS for fatty acid composition in backfat from pigs of two different breeds regarding fat level and distribution [9]. This allowed us to identify genomic loci associated with levels of de novo synthesized fatty acids. For the essential fatty acids, C18:2n-6, C18:3n-3 and total PUFA, no QTLs were detected in either of the breeds, which is expected as the level of PUFA is influenced mainly by diet [1].

The most significant QTL in this study was identified on SSC14 in Duroc where it explains up to $76 \%$ of the genetic variance and $55 \%$ of the phenotypic variance for the de novo synthesized fatty acids. This is slightly higher compared with a previous study of the same QTL showing up to $45 \%$ explained by genetic variance [25]. The QTL region from the GWAS is at 117.6-124.6 Mb and the fine mapping using sequence data narrowed down the peak to region $120-122 \mathrm{Mb}$. This QTL region has also previously been identified in subcutaneous fat and intramuscular fat of Duroc pigs at 120-124 Mb [16, 25] and in the longissimus dorsi muscle of different breeds and crossbreds $[15,20,21]$. SCD, located at 120.96$120.98 \mathrm{Mb}$ and encoding the main enzyme responsible for the desaturation of SFA to MUFA, is considered a very strong positional candidate gene for this QTL. SCD has been investigated as a candidate gene underlying fatty acid composition in pigs in several studies $[15,16$, $25,28-30$ ] and different haplotypes with $S C D$ variants have been found to be significant [30, 32]. After studying SNPs and haplotypes of SCD in different breeds, Estany and co-authors [28] suggested that the rs80912566 SNP, identified in Uemoto and co-authors [32], within the promoter region is the putative causative variant. This SNP is positioned in the core sequence of several putative transcription factor binding sites and was included in our fine mapping using sequence data. Results showed that it was among the highly associated SNPs but not the most significant one for either of the traits analyzed. The LD between $r s 80912566$ and the most significant SNPs detected by the GWAS was $r^{2}=0.80-0.95$, so our most significant SNPs would pick up the signal of rs80912566 if it is indeed the causal variant. We also examined the effect of the different homozygote genotypes of $r s 80912566$ on the actual phenotypic level of fatty acids, as done in Fig. 3 by the top SNPs. The difference between $\mathrm{AA}$ and $\mathrm{BB}$ for levels of $\mathrm{C} 16: 0$, expressed as \% of total fatty acids, was 1.16 for $r s 80912566$ and 1.43 for rs318243431. For C16:1, the difference was 0.5 for 


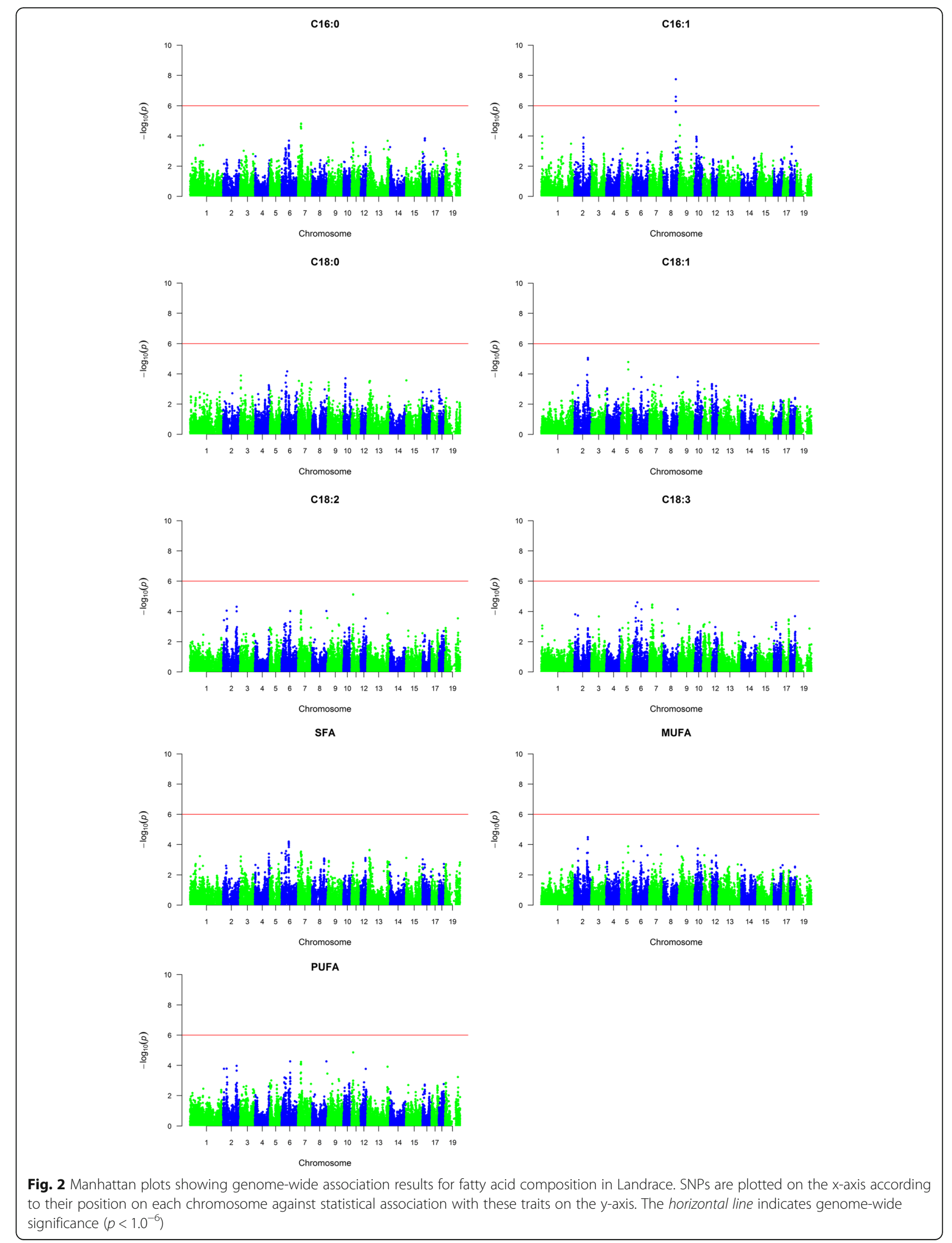


Table 3 The most significant SNP for each QTL and trait

\begin{tabular}{|c|c|c|c|c|c|c|c|c|}
\hline SSC & Trait & Breed & SNP & MAF & Allele subst. effect & $\% \sigma_{a}^{2}$ & $\% \sigma_{p}^{2}$ & Significance ( $p$-value) \\
\hline \multirow[t]{6}{*}{4} & C16:0 & Duroc & rs81241620 & 0.22 & 0.60 & 51 & 25 & $6.22 \mathrm{e}-14$ \\
\hline & C16:1n-7 & & rs323595907 & 0.22 & -0.24 & 60 & 34 & $4.27 \mathrm{e}-14$ \\
\hline & C18:0 & & rs323595907 & 0.22 & 1.82 & 67 & 48 & $3.40 \mathrm{e}-18$ \\
\hline & C18:1n-9 & & rs698347627 & 0.22 & -1.77 & 50 & 26 & $1.58 \mathrm{e}-11$ \\
\hline & SFA & & rs323595907 & 0.22 & 2.48 & 63 & 47 & $2.90 \mathrm{e}-19$ \\
\hline & MUFA & & rs698347627 & 0.22 & -2.25 & 57 & 30 & $8.05 e-13$ \\
\hline 8 & C16:1n-7 & Landrace & rs324018164 & 0.47 & -0.06 & 19 & 5 & $3.48 \mathrm{e}-08$ \\
\hline \multirow[t]{6}{*}{14} & C16:0 & Duroc & rs318243431 & 0.24 & 0.60 & 55 & 27 & $4.05 e-15$ \\
\hline & C16:1n-7 & & rs340458768 & 0.28 & -0.25 & 71 & 40 & $5.83 e-18$ \\
\hline & C18:0 & & rs318695446 & 0.24 & 1.91 & 76 & 55 & $1.63 e-21$ \\
\hline & C18:1n-9 & & rs318695446 & 0.24 & -1.82 & 55 & 28 & $2.00 \mathrm{e}-12$ \\
\hline & SFA & & rs318243431 & 0.24 & 2.55 & 71 & 54 & $3.16 \mathrm{e}-22$ \\
\hline & MUFA & & rs318695446 & 0.24 & -2.31 & 62 & 33 & $2.76 \mathrm{e}-14$ \\
\hline
\end{tabular}

For each QTL region and trait analyzed with the $660 \mathrm{~K}$ array, the most significant SNP is presented with ID, minor allele frequency (MAF), allele substitution effect, proportion of explained genetic variation $\left(\% \sigma_{\mathrm{a}}^{2}\right)$, proportion of explained phenotypic variation $\left(\% \sigma_{\mathrm{p}}^{2}\right)$ and significance ( $p$-value corrected for genomic control)

rs80912566 and 0.51 for rs340458768. Based on such marginal differences between our most significant SNP and the rs80912566 (SCD) we were not able to conclude which is the most likely causal variant.

Due to high LD in the QTL region on SSC14, it is impossible to conclude with specific causative genes or SNPs merely on fine mapping results. Positional candidate genes suggested from the GWAS positions obtained by $660 \mathrm{~K}$ and/or sequence data are SCD, CPN1, PAX2, PKD2L1, ENTPD7 and SEMA4G. The functions of PAX2, ENTPD7 and SEMA4G do not support a role in fatty acid composition, but the three other genes might be considered as biological interesting candidates. The protein encoded by CPN1 is the small subunit of carboxypeptidase $\mathrm{N}$, which is a metalloprotease that regulates peptide activity and receptor binding [55]. The carboxypeptidase member E protein expression has been linked to fatty acid levels in human [56], however, the biological function of carboxypeptidase member CPN1 is not fully understood [55]. PKD2L1 belongs to the polycystin family of transient receptor potential channel superfamily and it has previously been associated with levels of C16:1n-7 [57, 58] and the phospholipid C16:0 to C18:0 ratio [58]. However, the exact role of PKD2L1 is not known and the study by Wu et al. 2013 [57] suggested that $S C D$ was a better candidate gene due to its function. The thorough characterization of $S C D$ and its localization in the QTL peak makes it a very strong candidate gene for the association, however, from our results we cannot conclude that it is the causal one.

On SSC4, 21 significant SNPs were associated with the de novo synthesized fatty acids in Duroc at 63.8563.99 Mb. A QTL for fatty acid composition has previously been identified close to this QTL region, at $\sim 60 \mathrm{Mb}$ $[17,27]$. The SNP INRA0046679 at $63.8 \mathrm{Mb}$ on SSC4 in a study by Yang et al. [15] was significant for C18:0, however, the SNP was in complete LD with the most significant SNP on SSC14 ( 121 Mb) and the authors therefore concluded that this SNP is misplaced. In this study, the SNPs on SSC4 displayed high or complete LD $\left(r^{2}=0.85-\right.$ 1.0) with the most significant SNPs on SSC14. We therefore suspect, as was concluded in the study by Yang et al. [15], that the SNPs are misplaced on the marker map, probably due to errors in the reference genome [59].

In Landrace, we identified a QTL for C16:1n-7 on SSC8 with significant SNPs located between 120.5 and 121.4 Mb. Several previous studies have reported QTLs for fatty acid composition on this chromosome [13, 16-18, 21, 27]. One GWAS obtained a QTL overlapping the one we identified for the fatty acids C14:0, C16:1n-7 and C20:3 in backfat, as well as for $\mathrm{C} 16: 0$ and $\mathrm{C} 16: 1 \mathrm{n}-7$ in intramuscular fat [17]. The authors also identified genes surrounding this QTL region through eQTL analysis and the candidate gene closest to our most significant SNPs is ELOVL6, located at 120.1120.2 Mb. This gene encodes a fatty acid elongase that is involved in the elongation of C12-16 fatty acids to C18 [60]. Studies investigating polymorphisms in ELOVL6 in pig identified promoter SNPs associated with C16:0 and C16:1n-7 in backfat $[27,61]$ and suggested that a SNP located at -394 bp from the transcription start is a potential causative mutation [61]. This SNP is associated with an increased methylation level of the ELOVL6 promoter and decreased gene expression. The promoter SNP was found to explain $32 \%$ of the phenotypic variance for C16 in backfat of Iberian $x$ Landrace pigs [61] whereas the most significant SNP in our study, rs324018164, explained 19\% of the genetic variance and $5 \%$ of the phenotypic variance for $\mathrm{C} 16: 1 \mathrm{n}$ 7. Whether the ELOVL6 promoter SNP is the causative variant in Landrace needs to be further investigated. 

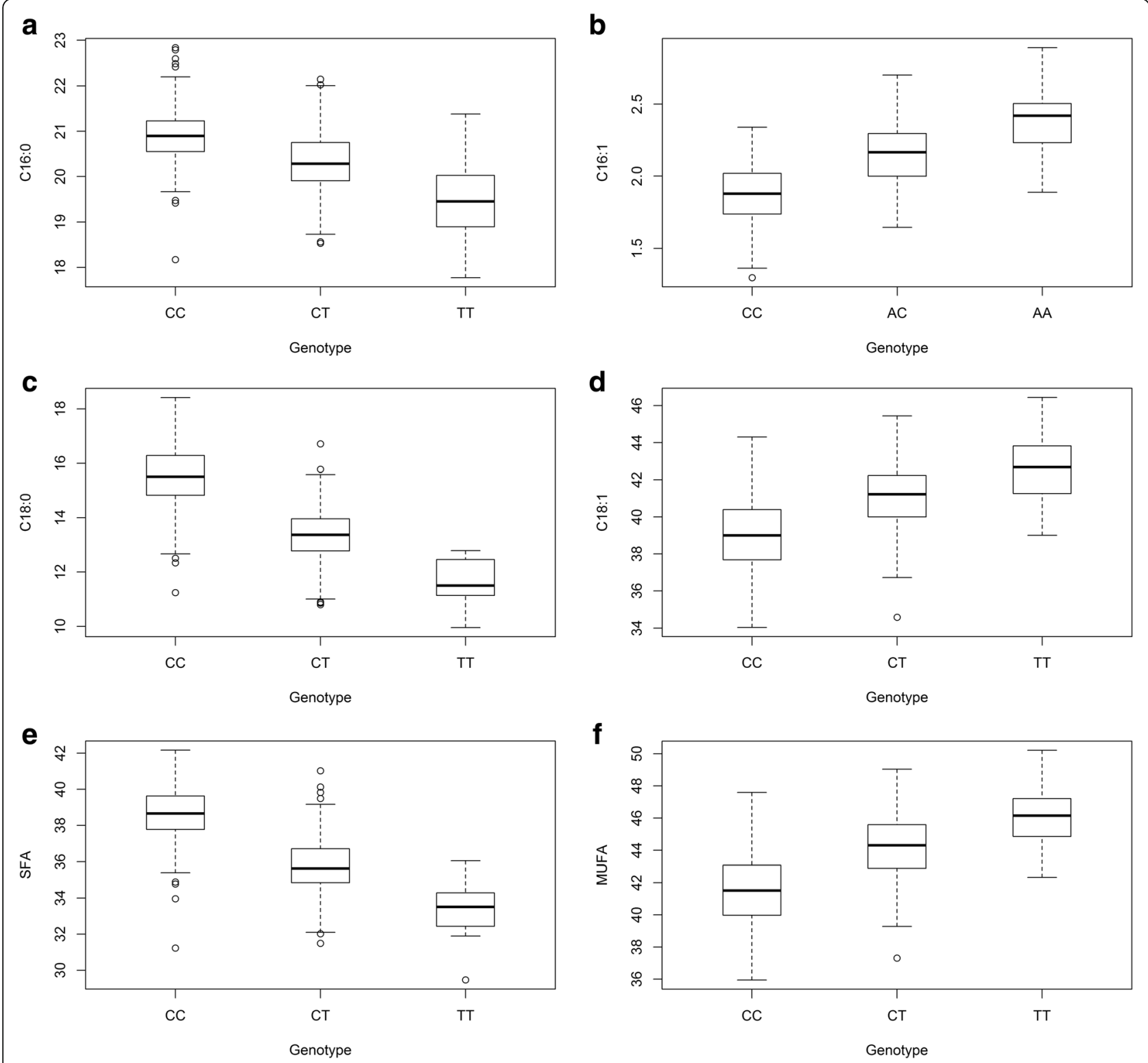

Fig. 3 Phenotypic mean per genotype class for SSC14 SNPs in Duroc. Box plots showing the differences in levels of fatty acids for the different genotypes of the most significant SNP for each trait of significance (rs318243431 for C16:0 and SFA, rs340458768 for C16:1n-7 and rs318695446 for C18:0, C18:1n-9 and MUFA). Box edges represent the upper and lower quartile with the median value shown as a bold line in the middle of the box. Whiskers represent 1.5 times the quartile of the data and individuals falling outside the range of the whiskers are shown as dots. a C16:0 b C16:1n-7 c C18:0 d C18:1n-9 e SFA f MUFA

It has been suggested that having high levels of C18:1n-9 in meat is favorable for technological quality and sensory properties, in addition to being favorable for human nutrition [1]. Fat from commercial pigs already contains $40-45 \% \mathrm{C} 18: 1 \mathrm{n}-9$, which is either de novo synthesized from carbohydrates in the diet or comes directly from the diet. Breeding for more $\mathrm{C} 18: 1 \mathrm{n}-9$ is possible since the trait is highly heritable $\left(h^{2}=0.60\right)[9,35]$, and now also by use of the QTL detected in this study. As shown in Fig. 3 the average phenotypic level will change from 39\% C18:1n-9 for the TT genotype of $r s 318695446$, to $41 \%$ for TC and $42.5 \%$ for CC, and the C18:0 will decrease accordingly. In general, oleic acid content is not a valued trait by meat producers and, consequently, not many breeding companies are focusing on fatty acid composition. However, it is an interesting trait in the particular case of traditional dry-cured ham and healthier pork products, and some breeding companies are exploring selecting for this trait. 


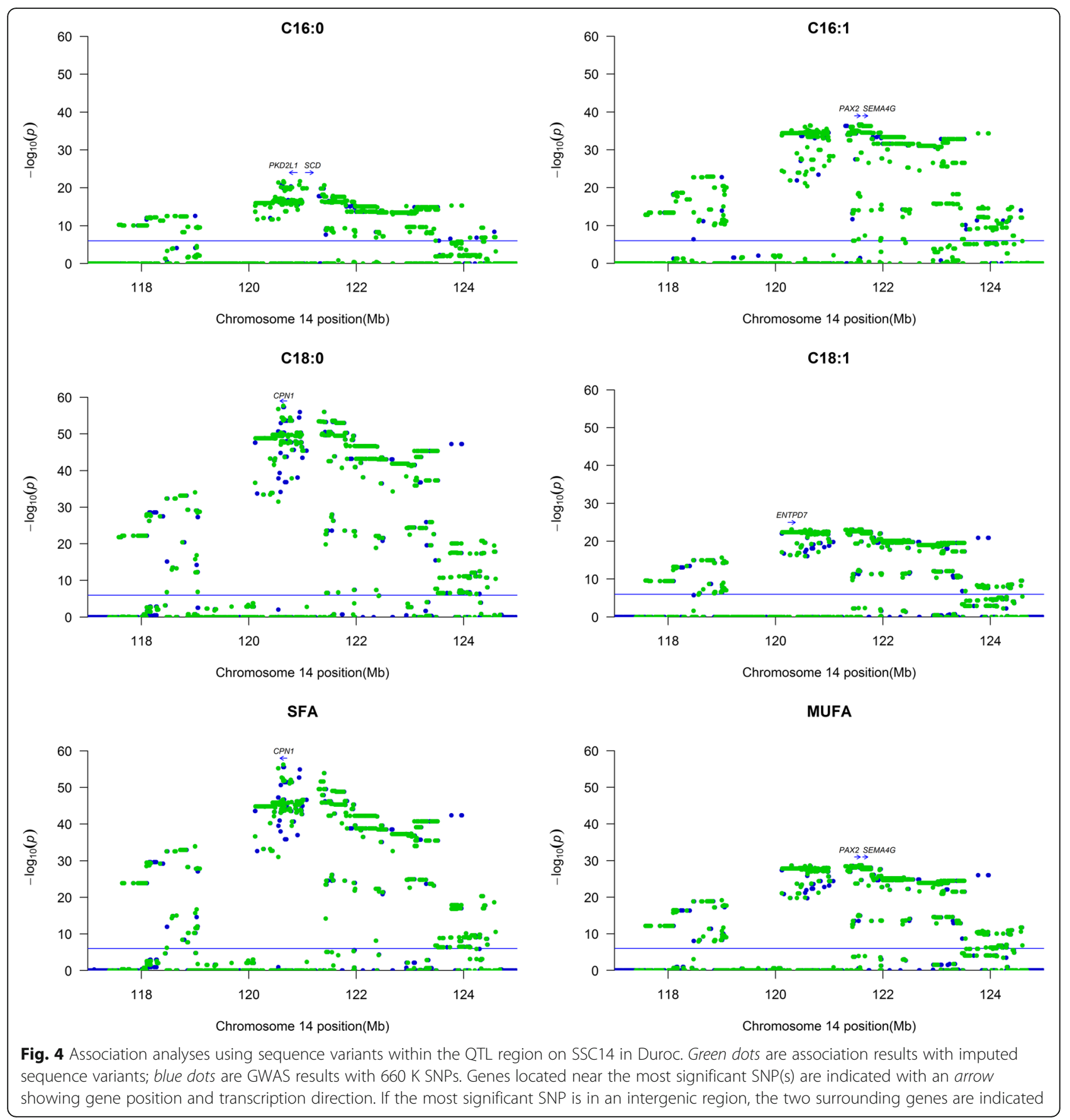

\section{Conclusions}

The present study confirms the importance of previously identified QTLs for fatty acid composition on SSC14 in Duroc pigs and on SSC8 in Landrace pigs. The most significant SNP in this study was found for Duroc on SSC14 and explained between 55 and $76 \%$ of the genetic variance and between 27 and 54\% of the phenotypic variance for the de novo synthesized fatty acid traits. Fine mapping of the QTL region on SSC14 confirmed the QTL but high LD made it difficult to identify causative variants. Based on our results the putative functional SNP suggested within $S C D$ could not be proven to be the causal one. In Landrace, a significant QTL was identified on SSC8 for C16:1n-7, explaining 19\% of the genetic variance and $5 \%$ of the phenotypic variance. The results of this study can be implemented in breeding to produce higher quality and healthier fatty acid composition of pork meat. 


\section{Additional files}

Additional file 1: Significant association statistics for the fatty acid traits in Duroc using the $660 \mathrm{~K}$ SNP array. For each trait, the significant SNPS are presented with SNP ID, chromosome and basepair position, and pvalue. (XLSX 5879 kb)

Additional file 2: Significant association statistics for the fatty acid traits in Landrace using the $660 \mathrm{~K}$ SNP array. For each trait, the significant SNPs are presented with SNP ID, chromosome and basepair position, and $p$ value. (XLSX $10 \mathrm{~kb}$ )

Additional file 3: Significant association statistics for the imputed sequence variants in the QTL region on SSC14 in Duroc. SNPS are presented with ID, chromosome, position and multiple testing adjusted $p$-values. (XLSX $297 \mathrm{~kb}$ )

\section{Abbreviations}

Al: Artificial insemination; CPN1: Carboxypeptidase N subunit 1; ELOVL6: ELOVL fatty acid elongase 6; ENTPD7: Ectonucleoside triphosphate diphosphohydrolase 7; FASTA: Family Based Score Test for Association; GLM: Generalized linear model; GWAS: Genome-wide association study; Indel: Insertion/deletion; LD: Linkage disequilibrium; MAF: Minor allele frequency; MTTP: Microsomal triglyceride transfer protein; MUFA: Monounsaturated fatty acids; NIRS: Near-infrared spectroscopy; PAX2: Paired box 2; PKD2L1: Polycystin 2 like 1, transient receptor potential cation channel; PUFA: Polyunsaturated fatty acids; QTL: Quantitative trait loci; SCD: Stearoyl-CoA desaturase; SEMA4G: Semaphoring 4G; SFA: Saturated fatty acids; SNP: Single nucleotide polymorphism; VEP: Variant Effect Predictor

\section{Acknowledgements}

The authors also wish to thank BioBank AS for sampling and DNA extraction, the Centre for Integrative Genetics (CIGENE) at the Norwegian University of Life Sciences for providing genotyping facilities, Hanne Hamland and Silje Karoliussen for genotyping the samples, and Juan Herrero Medrano, Marcos S. Lopes and Tim M. Knutsen for valuable input in data analysis.

\section{Funding}

This work was supported by the Research Council of Norway, Nortura SA and Norsvin SA. The funding bodies had no role in the design of the study or collection, analysis, and interpretation of data or in writing the manuscript.

\section{Availability of data and materials}

The newly detected and filtered SNPs from this study have been submitted to NCBI dbSNP. The raw data used during the current study are available from the corresponding author on request.

\section{Authors' contributions}

MvS conducted GWAS analyses, SNP detection and drafted the paper EGE was involved in project planning, organized the fatty acid measurements and contributed to writing the paper. HG conducted imputation work and took part in writing the paper. RRF contributed to fatty acid measurements and took part in writing the paper. MPK was involved in project planning, organized data for sequencing and contributed to writing the paper. SL was involved in project planning and contributed to writing the paper. EG was involved in project planning, provided experimental data and contributed to writing the paper. All authors read and approved the final manuscript.

\section{Competing interests}

The authors declare that they have no competing interests.

\section{Consent for publication}

Not applicable.

\section{Ethics approval}

The animals used in this study were raised at Norsvin's boar testing station. All animals were cared for according to laws, internationally recognized guidelines and regulations controlling experiments with live animals in Norway according to the rules given by the Norwegian Animal Research Authority (The Animal Protection Act of December $20^{\text {th }}$ 1974, and the Animal Protection Ordinance Concerning Experiments with Animals of January $\left.15^{\text {th }}, 1996\right)$. Ethics committee approval was not applicable for this study because the data were obtained from an existing database of pig breeding.

\section{Publisher's Note}

Springer Nature remains neutral with regard to jurisdictional claims in published maps and institutional affiliations.

\section{Author details}

${ }^{1}$ Norsvin SA, Storhamargata 44, 2317, Hamar, Norway. ${ }^{2}$ Centre for Integrative Genetics (CIGENE), Department for Animal and Aquacultural Sciences, Norwegian University of Life Sciences, P. O. Box 50031432 Ås, Norway. ${ }^{3}$ Departament de Ciència Animal, Universitat de Lleida-Agrotecnio Center, 191 Av Alcalde Rovira Roure, 25198 Lleida, Catalonia, Spain. ${ }^{4}$ Present address: The Roslin Institute and Royal (Dick) School of Veterinary Studies, The University of Edinburgh, EH25 9RG Midlothian, Scotland, UK.

Received: 23 January 2017 Accepted: 2 May 2017

Published online: 11 May 2017

\section{References}

1. Wood JD, Richardson RI, Nute GR, Fisher AV, Campo MM, Kasapidou E, Sheard PR, Enser M. Effects of fatty acids on meat quality: a review. Meat Sci. 2004;66(1):21-32.

2. Wood JD, Enser M, Fisher AV, Nute GR, Sheard PR, Richardson RI, Hughes SI, Whittington FM. Fat deposition, fatty acid composition and meat quality: a review. Meat Sci. 2008;78(4):343-58.

3. Kris-Etherton PM. Monounsaturated fatty acids and risk of cardiovascular disease. Circulation. 1999;100:1253-8.

4. Schwingshackl L, Hoffmann G. Monounsaturated fatty acids, olive oil and health status: a systematic review and meta-analysis of cohort studies. Lipids Health Dis. 2014;13:154.

5. Chowdhury R, Warnakula S, Kunutsor S, Crowe F, Ward HA, Johnson L, Franco OH, Butterworth AS, Forouhi NG, Thompson SG, et al. Association of dietary, circulating, and supplement fatty acid with coronary risk: a systematic review and meta-analysis. Ann Intern Med. 2014;160(6):398-406.

6. de Souza RJ, Mente A, Maroleanu A, Cozma Al, Ha V, Kishibe T, Uleryk E, Budylowski P, Schünemann $H$, Beyene J, et al. Intake of saturated and trans unsaturated fatty acids and risk of all cause mortality, cardiovascular disease, and type 2 diabetes: systematic review and meta-analysis of observational studies. BMJ. 2015;351:h3978.

7. Cameron ND, Enser M, Nute GR, Whittington FM, Penman JC, Fisken AC, Perry AM, Wood JD. Genotype with nutrition interaction on fatty acid composition of intramuscular fat and the relationship with flavour of pig meat. Meat Sci. 2000;55(2):187-95.

8. Ruiz-Carrascal J, Ventanas J, Cava R, Andrès Al, Garcia C. Texture and appearance of dry cured ham as affected by fat content and fatty acid composition. Food Res Int. 2000:33(2):91-5.

9. Gjerlaug-Enger E, Aass L, Ødegård J, Kongsro J, Vangen O. Genetic parameters of fat quality in pigs measured by near-infrared spectroscopy. Animal. 2011;5(10):1495-505.

10. Fernandez A, de Pedro E, Núñez N, Silió L, García-Casco J, Rodríguez C. Genetic parameters for meat and fat quality and carcass composition traits in Iberian pigs. Meat Sci. 2003;64(4):405-10.

11. Sellier P. Genetics of meat and carcass traits. In: Rothschild MF, Ruvinsky A, editors. The genetics of the pig. New York: CAB International; 1998. p. 463-510.

12. Pérez-Enciso M, Clop A, Noguera JL, Ovilo C, Coll A, Folch JM, Babot D, Estany J, Oliver MA, Díaz I, et al. A QTL on pig chromosome 4 affects fatty acid metabolism: evidence from an Iberian by Landrace intercross. J Anim Sci. 2000;78(10):2525-31.

13. Guo T, Ren J, Yang K, Ma J, Zhang Z, Huang L. Quantitative trait loci for fatty acid composition in longissimus dorsi and abdominal fat: results from a White Duroc $x$ Erhualian intercross $F_{2}$ population. Anim Genet. 2009;40(2):185-91.

14. Nii M, Hayashi T, Tani F, Niki A, Mori N, Fujishima-Kanaya N, Komatsu M, Aikawa K, Awata T, Mikawa S. Quantitative trait loci mapping for fatty acid composition traits in perirenal and back fat using a Japanese wild boar $\mathrm{x}$ Large White intercross. Anim Genet. 2006;37(4):342-7.

15. Yang B, Zhang W, Zhang Z, Fan Y, Xie X, Ai H, Ma J, Xiao S, Huang L, Ren J. Genome-wide association analyses for fatty acid composition in porcine muscle and abdominal fat tissues. PLoS One. 2013;8(6):e65554. 
16. Uemoto Y, Soma Y, Sato S, Ishida M, Shibata T, Kadowaki H, Kobayashi E, Suzuki K. Genome-wide mapping for fatty acid composition and melting point of fat in a purebred Duroc pig population. Anim Genet. 2011:43:27-34.

17. Muñoz M, Rodríguez MC, Alves E, Folch JM, Ibañez-Eschriche N, Silió L, Fernández Al. Genome-wide analysis of porcine backfat and intramuscular fat fatty acid composition using high-density genotyping and expression data. BMC Genomics. 2013;14:845

18. Clop A, Ovilo C, Perez-Enciso M, Cercos A, Tomas A, Fernandez A, Coll A, Folch JM, Barragan C, Diaz I, et al. Detection of QTL affecting fatty acid composition in the pig. Mamm Genome. 2003;14:650-6.

19. Ramayo-Caldas Y, Ballester M, Fortes MRS, Esteve-Codina A, Castelló A, Noguera JL, Fernández Al, Pérez-Enciso M, Reverter A, Folch JM. From SNP co-association to RNA co-expression: novel insights into gene networks for intramuscular fatty acid composition in porcine. BMC Genomics. 2014;15:232.

20. Sanchez M-P, lannucelli N, Basso B, Bidanel JP, Billon Y, Gandemer G, Gilbert H, Larzul C, Legault C, Riguet J, et al. Identification of QTL with effects on intramuscular fat content and fatty acid composition in a Duroc $x$ Large White cross. BMC Genet. 2007;8:55.

21. Zhang W, Zhang J, Cui L, Ma J, Chen C, Ai H, Xie X, Li L, Xiao S, Huang L, et al. Genetic architecture of fatty acid composition in the longissimus dorsi muscle revealed by genome-wide association studies on diverse pig populations. Genet Sel Evol. 2016;48:5

22. Ramayo-Caldas Y, Mercadé A, Castelló A, Yang B, Rodríguez C, Alves E, Díaz I, Ibañez-Eschriche N, Noguera JL, Pérez-Enciso M, et al. Genome-wide association study for intramuscular fatty acid composition in an Iberian $\mathrm{x}$ Landrace cross. J Anim Sci. 2012;90(9):2883-93.

23. Kim $Y$, Kong M, Nam YJ, Lee C. A quantitative trait locus for oleic fatty acid content on Sus scrofa chromosome 7. J Hered. 2006;97(5): 535-7.

24. Uemoto $Y$, Sato S, Ohnishi C, Terai S, Komatsuda A, Kobayashi E. The effects of single and epistatic quantitative trait loci for fatty acid composition in a Meishan $x$ Duroc crossbred population. J Anim Sci. 2009;87(11):3470-6

25. Ros-Freixedes R, Gol S, Pena RN, Tor M, Ibañez-Eschriche N, Dekkers JCM, Estany J. Genome-wide association study singles out SCD and LEPR as the two main loci influencing intramuscular fat content and fatty acid composition in Duroc pigs. PLoS One. 2016;11(3):e0152496.

26. Estellé J, Fernández Al, Pérez-Enciso M, Fernandez A, Rodríguez C, Sánchez A, Noguera JL, Folch JM. A non-synonymous mutation in a conserved site of the MTTP gene is strongly associated with protein activity and fatty acid profile in pigs. Anim Genet. 2009;40(6):813-20.

27. Corominas J, Ramayo-Caldas Y, Puig-Oliveras A, Pérez-Montarelo D, Noguera JL, Folch JM, Ballester M. Polymorphism in the ELOVL6 gene is associated with a major QTL effect on fatty acid composition in pigs. PLoS One. 2013;8(1):e53687

28. Estany J, Ros-Freixedes R, Tor M, Pena RN. A functional variant in the Stearoyl-CoA desaturase gene promoter enhances fatty acid desaturation in pork. PLoS One. 2014;9(1):e86177.

29. Lim K-S, Kim J-M, Lee E-A, Choe J-H, Hong K-C. A candidate single nucleotide polymorphism in the 3 'Untranslated region of Stearoyl-CoA desaturase gene for fatness quality and the gene expression in Berkshire pigs. Asian Australas J Anim Sci. 2015;28(2):151-7.

30. Maharani D, Park H-B, Lee J-B, Yoo C-K, Lim H-T, Han S-H, Lee S-S, Ko M-S, Cho I-C, Lee J-H. Association of the gene encoding stearoyl-CoA desaturase (SCD) with fatty acid composition in an intercross population between Landrace and Korean native pigs. Mol Biol Rep. 2013;40:73-80.

31. Mercadé A, Estellé J, Pérez-Enciso M, Varona L, Silió L, Noguera JL, Sánchez A, Folch JM. Characterization of the porcine acyl-CoA synthetase long-chain 4 gene and its association with growth and meat quality traits. Anim Genet. 2006;37(3):219-24.

32. Uemoto $Y$, Nakano H, Kikuchi T, Sato S, Ishida M, Shibata T, Kadowaki H, Kobayashi E, Suzuki K. Fine mapping of porcine SSC14 QTL and SCD gene effects on fatty acid composition and melting point of fat in a Duroc purebred population. Anim Genet. 2011;43:225-8.

33. Gjerlaug-Enger $E$, Aass L, Ødegård J, Vangen O. Genetic parameters of meat quality traits in two pig breeds measured by rapid methods. Animal. 2010; 4(11):1832-43

34. De Pedro E, Casillas M, Miranda CM. Microwave over application in the extraction of fat from the subcutaneous tissue of Iberian pig ham. Meat Sci. 1997:45:45-51.
35. Gjerlaug-Enger E, Kongsro J, Aass L, Ødegård J, Vangen O. Prediction of fat quality in pig carcasses by near-infrared spectroscopy. Animal. 2011; 5(11):1829-41.

36. Groenen MAM. Development of a high-density Axiom ${ }^{\circledast}$ porcine genotyping array to meet research and commercial needs. In: Plant \& Animal Genome XXIII Conference: 2015; San Diego, CA, USA. 2015.

37. Ramos AM, Crooijmans RPMA, Affara NA, Amaral AJ, Archibald AL, Beever JE, Bendixen C, Churcher C, Clark R, Dehais P. Design of a high density SNP genotyping assay in the pig using SNPs Identified and characterized by next generation sequencing technology. PLoS One. 2009;4(8):e6524.

38. Groenen MA, Archibald AL, Uenishi H, Tuggle CK, Takeuchi Y, Rothschild MF Rogel-Gaillard C, Park C, Milan D, Megens HJ, et al. Analysis of pig genomes provide insight into porcine demography and evolution. Nature. 2012; 491(7424):393-8.

39. Sargolzaei M, Chesnais JP, Schenkel FS. A new approach for efficient genotype imputation using information from relatives. BMC Genomics. 2014;15:478

40. Aulchenko YS, Ripke S, Isaacs A, van Duijn CM. GenABEL: an R library for genome-wide association analysis. Bioinformatics. 2007;23(10):1294-6.

41. R: A language and environment for statistical computing. [https://www. R-project.org]

42. Turner SD. An R package for visualizing GWAS results using Q-Q and manhattan plots. biorXiv. 2014; doi:10.1101/005165.

43. Gilmour AR, Cullis BR, Gogel BJ, Welham SJ, Thompson R. ASReml User Guide Release 3.0. UK: VSN International Ltd; 2009.

44. Falconer DS, Mackay TFC. Introduction to quantitative genetics. Harlow: Longmans Green; 1996.

45. Barrett JC, Fry B, Maller J, Daly MJ. Haploview: analysis and visualization of LD and haplotype maps. Bioinformatics. 2005;21(2):263-5.

46. Joshi NA, Fass JN. Sickle: a sliding-window, adaptive, quality-based trimming tool for FastQ files (version 1.33). 2011. Available at https:// github.com/najoshi/sickle.

47. Li H, Durbin R. Fast and accurate short read alignment with BurrowsWheeler transform. Bioinformatics. 2009;25:1754-60.

48. Li H, Handsaker B, Wysoker A, Fennell T, Ruan J, Homer N, Marth G, Abecasis G, Durbin R, Subgroup GPDP. The sequence alignment/map (SAM) format and SAMtools. Bioinformatics. 2009;25:2078-9.

49. Garrison E, Marth G. Haplotype-based variant detection from short-read sequencing. 2012. arXiv:12073907.

50. Danecek P, Auton A, Abecasis G, Albers CA, Banks E, DePristo MA, Handsaker RE, Lunter G, Marth GT, Sherry ST, et al. The variant call format and VCFtools. Bioinformatics. 2011;27(15):2156-8.

51. McLaren W, Pritchard B, Rios D, Chen Y, Flicek P, Cunningham F. Deriving the consequences of genomic variants within the Ensembl API and SNP Effect Predictor. Bioinformatics. 2010;26(16):2069-70.

52. Browning BL, Browning SR. Genotype imputation with millions of reference samples. Am J Hum Genet. 2016;98:116-26.

53. Browning SR, Browning BL. Rapid and accurate haplotype phasing and missing data inference for whole genome association studies using localized haplotype clustering. Am J Hum Genet. 2007:81:1084-97.

54. Sherry ST, Ward MH, Kholodov M, Baker J, Phan L, Smigielski EM, Sirotkin K. dbSNP: the NCBI database of genetic variation. Nucleic Acids Res. 2001; 29(1):308-11.

55. Matthews KW, Mueller-Oritz SL, Wetsel RA. Carboxypeptidase N: a pleiotropic regulator of inflammation. Mol Immunol. 2004;40:785-93.

56. Jeffrey KD, Alejandro EU, Luciani DS, Kalynyak TB, Hu X, Li H, Lin Y, Townsend BR, Polonsky KS, Johnson JD. Carboxypeptidase E mediates palmitate-induced $\beta$-cell ER stress and apoptosis. Proc Natl Acad Sci U S A 2008;105:24

57. Wu JH, Lemaitre RN, Manichaikul A, Guan W, Tanaka T, Foy M, Kabagambe EK, Djousse L, Siscovick D, Fretts AM, et al. Genome-wide association study identifies novel loci associated with concentrations of four plasma phospholipid fatty acids in the de novo lipogenesis pathway: results from the Cohorts for Heart and Aging Research in Genomic Epidemiology (CHARGE) consortium. Circ Cardiovasc Genet. 2013:6(2):171-83.

58. Demirkan A, Van Duijn CM, Ugocsai P, Isaacs A, Pramstaller PP, Liebisch G, Wilson JF, Johansson $\AA$, Rudan I, Aulchenko YS, et al Genome-wide association study identifies novel loci associated with circulating phospho- and sphingolipid concentrations. PLoS Genet. 2012;8(2):e1002490 
59. Warr A, Robert C, Hume D, Archibald AL, Deeb N, Watson M. Identification of low-confidence regions in the pig reference genome (Sscrofa10.2). Front Genet. 2015;6:338.

60. Jakobsson A, Westerberg $R$, Jacobsson A. Fatty acid elongases in mammals: their regulation and roles in metabolism. Prog Lipid Res. 2006;45(3):237-49.

61. Corominas J, Marchesi JAP, Puig-Oliveras A, Revilla M, Estellé J, Alves E, Folch

$J \mathrm{M}$, Ballester M. Epigenetic regulation of the ELOVL6 gene is associated with a major QTL effect on fatty acid composition in pigs. Genet Sel Evol. 2015;47:20.

Submit your next manuscript to BioMed Central and we will help you at every step:

- We accept pre-submission inquiries

- Our selector tool helps you to find the most relevant journal

- We provide round the clock customer support

- Convenient online submission

- Thorough peer review

- Inclusion in PubMed and all major indexing services

- Maximum visibility for your research

Submit your manuscript at www.biomedcentral.com/submit 